\title{
Identidad de género y empoderamiento femenino en las habitantes de la asociación de vecinos Quinta Elena, 2020
}

\author{
Gender identity and female empowerment in the inhabitants of the Quinta Elena \\ Neighborhood Association, 2020
}

\author{
Heidy Cristina Navas Gómez ${ }^{1 \mathrm{a}}$, Ana Belén Malpartida Portocarrero, ${ }^{2}$ \& \\ Renzo Felipe Carranza Esteban ${ }^{3}$ \\ Universidad Peruana Unión, filial Tarapoto, Perú ${ }^{123}$ \\ ORCID ID: 0000-0002-4015-1650 1 \\ ORCID ID: 0000-0001-7999-3786² \\ ORCID ID: $0000-0002-4086-4845^{3}$
}

Recibido: 12 de octubre 2020

Aceptado: 26 de marzo de 2021

\section{Resumen}

El presente estudio tuvo como objetivo determinar si existe relación significativa entre la identidad de género y el empoderamiento femenino entre las habitantes de la asociación de vecinos Quinta Elena 2020, se usó una investigación aplicada, cuantitativa, con diseño no experimentalcorrelacional de corte transversal, estando la muestra constituida por 120 mujeres de la asociación de vecinos Quinta Elena, se aplicó el Cuestionario de actitudes hacia la identidad de género (CAIG)- Modificado y el Instrumento para la medición del empoderamiento en mujeres (IMEN). Se observó que la identidad de género presentó un nivel medio en $45.0 \%$ y el empoderamiento también tuvo un nivel medio en $74.2 \%$, en tanto, se tuvo como conclusión que existe relación entre las variables evaluadas ( $<<.05 ; \mathrm{r}=.747)$, confirmando la hipótesis planteada.

Palabras clave: Identidad de género, empoderamiento, autoconcepto, roles de género.

\begin{abstract}
The present study aimed to determine if there is a significant relationship between gender identity and female empowerment among the inhabitants of the residents of the Quinta Elena 2020 neighborhood association, an applied, quantitative research with a non-experimental-correlational design was used. cross-sectional section, with the sample consisting of 120 women from the Quinta Elena neighborhood association, the instrument "Questionnaire on attitudes towards gender identity (CAIG) - Modified” and the "Instrument for measuring empowerment in women (IMEN)".
\end{abstract}


Finally, it was observed that gender identity is medium in $45.0 \%$, in the same way, empowerment also registered a medium level in $74.2 \%$, reaching the conclusion that there is a relationship between the variables evaluated $(p<.05 ; r=.747)$ which demonstrates the proposed hypothesis.

Keywords: Gender identity, empowerment, self-concept, gender roles.

\section{Introducción}

En el Perú, según la Agencia Peruana de Noticias (2018), se ha visto una mejora en el índice global de brecha de género posicionándose en el puesto 52, donde tuvo lugar una disminución de las diferencias entre hombres y mujeres; de igual manera, El Ministerio de la Mujer y Poblaciones Vulnerables se encuentra trabajando para que el país llegue a una equidad de género total (Torres, 2019). A pesar de desarrollarse programas como el “Empoderar para Incluir”, aun se aprecia un camino largo que recorrer (Ruíz, Vargas y Clausen, 2018). Durante el 2020, a pesar de las situaciones en la que se ha venido desarrollando como el bajo nivel de consideración de las mujeres a nivel organizacional o promocional, según el reporte presentado por MasterCard el país ocupa el puesto 3 de empoderamiento a nivel de América Latina (Fundación Romero, 2020).

El empoderamiento de la mujer ha trascendido y tuvo gran importancia para el desarrollo de la sociedad actual hacia una colectividad más equitativa (Urzelai, 2014). Sin embargo, en la Asociación de Vecinos Quinta Elena, perteneciente al distrito de Banda de Shilcayo, se evidenció mediante una entrevista que las habitantes no tienen conocimientos suficientes sobre la identidad de género, es decir; al sujeto mujer- femenino, se le asignan tareas que se desarrollan en el ámbito doméstico y cuyos fines se fundamentan en la reproducción biológica, la crianza y los cuidados, la cual no están valoradas ni económicamente (pues no se percibe remuneración por ellas), ni culturalmente (pues no se habla de ellas, son invisibles), ni socialmente (pues se desarrollan en espacios cerrados y aislados -el hogar-).

Efectuando un análisis de información que contribuyen al reforzamiento de la investigación, se obtuvo en México el estudio planteado por Duarte y García (2016), quienes en sus resultados evidenciaron que desde hace muchos años atrás el rol de los hombres es sobrevalorado, mientras que el rol de las mujeres pasa desapercibido; por su parte Farías y Cuello (2018), reconocieron que la publicidad tiene un impacto significativo en las percepciones de los jóvenes sobre la igualdad de género, por cuanto proyectan un comportamiento machista en donde 
el varón asume un papel más importante que la mujer. En España, Ros y Lidón (2018), encontraron que la experiencia del empoderamiento en mujeres mayores de 65 años fue precaria, por ejemplo, en la toma de decisiones indicaron que el 40\% sentía que no podía tomarlos por cuenta propia, mientras que el $40 \%$ sentía que su opinión era valiosa y el $40 \%$ aseguraba que sus decisiones no eran significativas.

A nivel nacional, Espinoza (2020), manifiesta en su estudio que las redes sociales influyen de manera significativa en la conducta y pensamiento de las personas, es decir centrado en la población analizada, la percepción de empoderamiento se ve afectada significativamente; Alvarado (2016), señala que en épocas antiguas las mujeres no gozaban de respeto y admiración debido a que no ejercían libremente los derechos que les correspondían suscitando que no sean partícipes de los acontecimientos políticos, sociales, religiosos; por su parte, Calderón (2017), en un estudio a mujeres indígenas, identificó que el proceso de empoderamiento está asociado al espacio organizacional que funciona como un impulsor del cambio, por otro lado, en cuanto a la identidad de género, en su mayoría estas mujeres no compartían los mismos roles con su pareja; Boza (2019), identifico en una organización de Club de Madres en la ciudad de Ayacucho que el 68.6\% de las mujeres encuestadas tenían un nivel promedio de empoderamiento y el 22.9\% un nivel alto, y en relación al sentido de igualdad en el género, el 69.0\% presentó un nivel promedio de igualdad, y el $26.0 \%$ un nivel alto.

Respecto al conocimiento teórico del constructo identidad de género, diferentes autores realizan una definición sobre esta, Ramos (1999), señala que la identidad de género es una de las manifestaciones externas de los diversos rasgos culturales que permiten a los sujetos identificarse con un rol según los patrones de conducta considerados como propios para cada género, en un tiempo, momento y espacio respectivo; Gauché y Lovera (2018), expresan que la identidad de género es una identidad que se adquiere mediante el aprendizaje a lo largo de los primeros años de vida, la construcción de la identidad de género forma parte de la vivencia interna e individual que cada sujeto experimenta por sí mismo; para Rocha (2009), la identidad de género refiere a una construcción sobre el ser hombre o mujer, siendo este un proceso de con cierto nivel de complejidad puesto que comprende la interacción de los factores biológicos, sociales, culturales y psicológicos.

Existen diversos modelos teóricos psicológicos que explican la formación de la identidad de género, desde el enfoque psicodinámico se tiene la explicación realizada por Freud (1957), quien señala que, la formación de la identidad genérica ocurre por medio de las interacciones que tiene 
el infante con el cuidador primario, es durante la infancia cuando el niño o niña interioriza la visión, características y los roles del cuidador, sirviendo de bases para su estructura psíquica. Desde el enfoque de aprendizaje y aprendizaje social se da mucho énfasis a la comunicación que es la que posibilita el desarrollo cognitivo y la capacidad de aprender como elementos centrales que explican la formación de la identidad de género, es decir, que un individuo aprende a ser hombre o mujer por medio de la comunicación y la observación, imitando a las personas adultas, padres, amigos, figuras de la televisión, y todo medio posible de aprendizaje, es así que con el tiempo el sujeto va construyendo su propia identidad de género (Bandura y Walters, 1963; Lynn, 1965, Mischel, 1966).

Por último, se tiene al modelo teórico multifactorial de la identidad de género, que explica la complejidad en la que se forma este constructo, aludiendo a un proceso constante y permanente de socialización a lo largo de la vida, donde se interiorizan los estereotipos y roles que se les asignan a nombres y mujeres culturalmente y canalizándolos mediante la ejecución de conductas diferenciales y la adopción de características distintivas según el género, según esta teoría, entran en juego diversas variables como la parte cognitiva, social, cultural, biológica y psicológica (Rocha, 2009).

En relación al empoderamiento femenino, se tiene a distintos autores que conceptualizan el término, para Ruiz, Turnbull y Cruz (2016), el empoderamiento femenino hace referencia a la capacidad de toma de decisiones que posee una mujer, tales decisiones promueven su autonomía y le hacen sentir que tiene dominio y poder sobre su propia vida; para Charlier et al. (2007), este concepto se define como la acción de tomar el poder, refiriéndose al fortalecimiento del autoestima y la confianza de la mujer, la capacidad de elegir el rumbo de su destino, además, se lo relaciona con el poder colectivo para cambiar las relaciones de género en distintos ámbitos de la vida, como el área económica, jurídica, sociocultural y política; por otro lado, para Casique (2006), el empoderamiento femenino alude a cuestiones de poder en las decisiones del hogar, el acceso al manejo de los recursos del hogar, el poder de decisión sobre las relaciones sexuales, la contribución de los ingresos económicos en la familia, tener participación política, conocer sus derechos, etc.

\section{Método}

De acuerdo con Ñaupas et al. (2014) el tipo de investigación fue aplicada, puesto que tiene propósitos prácticos inmediatos bien definidos, es decir, se investigó para actuar, trasformar, modificar o producir cambios en un determinado sector de la realidad, de igual manera fue 
cuantitativa, por cuanto busca la contrastación mediante procesos estadístico, además, el diseño fue no experimental, y según estos mismos autores esto se fundamenta en la utilización de una serie de símbolo que tienen una denotación que es importante conocer para leerlos comprensivamente, presenta un diseño correlacional, donde las variables de estudio fueron analizadas para encontrar los niveles de relación, contando además con un diseño de corte transversal, evaluados en una serie de tiempo concreto.

La población femenina fue de 250 mujeres de los cuales se consideró una muestra conformada por un total de 120 mujeres consideradas habitantes en la asociación de vecinos Quinta Elena, del distrito de la Banda de Shilcayo, Región San Martín, año 2020. En el desarrollo del estudio se consideró un muestreo no probabilístico, debido a que las participantes fueron elegidas por los investigadores sin aplicar criterios estadísticos para su ubicación, esto permitió el recojo de información más apropiado (Otzen \& Mantareola, 2017).

Para la variable identidad de género se consideró utilizar como instrumento el Cuestionario de actitudes hacia la igualdad de género (ACTIG), elaborado por García et al. (2010), cuyo propósito es medir las actitudes hacia la igualdad de género; el cuestionario está compuesto de tres dimensiones: Sociocultural (ítems del 1 al 10), relacional (ítems del 11 al 20), personal (ítems del 21 al 30); cada dimensión consta de 6 ítems, haciendo un total de 18 ítems, las opciones de respuesta son de tipo Likert, entre las que se encuentran: Totalmente en desacuerdo (1), en desacuerdo (2), ni de acuerdo ni en desacuerdo (3), de acuerdo (4), y totalmente de acuerdo (5). La confiabilidad del instrumento ha sido medida con el coeficiente de alfa de Cronbach obteniendo un valor de $\alpha=.91$, y para la validez de constructo la escala presenta unidimensionalidad con altos índices de saturación de todos los ítems en el componente principal, siendo la media un valor de 0,525 con una desviación típica de 0.088; además, para la validez de contenido se sometió a juicio de expertos, posterior a su evaluación se realizó un análisis ítem por ítem obteniendo un valor p>90, ubicándolo en un nivel muy bueno.

Para la variable empoderamiento femenino se consideró oportuno emplear el Instrumento para Medir el Empoderamiento de la Mujer, elaborado por Hernández y García (2008), este cuestionario tiene como objetivo de determinar el nivel de empoderamiento de las mujeres. Está compuesto de 7 factores, entre ellos: empoderamiento participativo (ítems 10, 8, 33, 9, 34, 32 y 20), temeridad, (ítems 4, 19, 5, 18 y 11), influencias externas, (ítems 6, 17, 7, 13, 12 у 16), independencia (ítems 1, 15, 29 y 2), igualdad (ítems 14, 3, 28 y 30), satisfacción social (ítems 31, 
26, 23 y 27) y seguridad, (ítems 24, 22, 25 y 21), con un total de 34 ítems, las opciones de respuesta son de tipo Likert, con cuatro opciones: totalmente en desacuerdo (1), en desacuerdo pero no totalmente (2), de acuerdo pero no totalmente (3), y totalmente de acuerdo (4). Cabe precisar que para la confiabilidad de los instrumentos se empleó el coeficiente de alfa de Cronbach y omega de McDonald, obteniendo un valor de $\alpha=.84 ; \Omega=.84$ respectivamente, por otro lado, para la validez de constructo se realizó el análisis factorial Varimax, donde se reportó que 7 factores explican el 54.72\% de la varianza total, así también para la validez de contenido se sometió a un análisis de juicio de cinco expertos y mediante el análisis ítem por ítem, donde se obtuvo un valor p>0.92, posicionándote en un nivel excelente.

En la investigación se elaboró una base de datos para ambas variables, con el propósito de agilizar el análisis de la información y garantizar su posterior uso o interpretación; seguidamente hizo uso del programa estadístico SPSS versión 26, dado que emplearon tablas de frecuencia, contingencia, tablas estadísticas, para obtener las frecuencias totales obtenidas en la tabulación de los datos referentes a las dimensiones de las varíales; es decir un análisis inferencial; cuando se tuvieron todos los permisos pertinentes, se procedió a efectuar la aplicación de los instrumentos de manera presencial, luego, los datos fueron ingresaron en el programa Excel para su posterior registro en el SPSS, que permitió el análisis de datos que se ubican en el apartado siguiente.

\section{Resultados}

De un análisis sociodemográfico se determinó una predominancia de edad comprendidas entre los 18-25 con el 43.3\% y de 26-35 años de 30.0\%, con un nivel de educación secundaria del 47.5\% y superior con el 39.2\%; en relación al estado civil, el 35.0\% fueron convivientes, el 28.3\% casados, y el 27.5\% solteros; finalmente, respecto a número de hijos, el 26,7\% señalaron que no tenían ningún hijo, el 19.2\% señalaron tener 3 hijos, y el 28.3\% 2 hijos (Ver tabla 1).

Tabla 1

Datos demográficos

\begin{tabular}{lll}
\hline Categoría & $F$ & $\%$ \\
\hline Edad & & \\
\hline$(18-25)$ & 52 & 43.3 \\
$(26-35)$ & 36 & 30.0 \\
(36-más) & 32 & 26.7 \\
\hline
\end{tabular}




\begin{tabular}{lcc}
\hline Nivel de educación & & \\
\hline Primaria & 16 & 13.3 \\
Secundaria & 57 & 47.5 \\
Superior & 47 & 39.2 \\
\hline Estado civil & & \\
\hline Soltera & 33 & 27.5 \\
Casada & 34 & 28.3 \\
Viuda & 7 & 5.8 \\
Divorciada & 4 & 3.3 \\
Conviviente & 42 & 35.0 \\
\hline Número de hijos & & \\
\hline Ninguno & 32 & 26.7 \\
1 & 20 & 16.7 \\
2 & 22 & 18.3 \\
3 & 23 & 19.2 \\
De 4 a más & 23 & 21.1 \\
\hline Total & 120 & 100.0 \\
\hline
\end{tabular}

Nota: Aplicación del SPSS26

De acuerdo con un análisis de los instrumentos aplicados se observó que la identidad de género en el $45.0 \%$ se ubicó en un nivel medio, en la categoría adaptativa, de este resultado se interpreta que, la mayoría de participantes tienen una flexibilidad en cuanto a sus actitudes hacia las cuestiones de género, con lo cual, pueden mostrarse en a favor de la igualdad de género o no, dependiendo de las circunstancias en las que se encuentren, por otro lado, el 30.0\% de la muestra se ubicó en un nivel alto en cuando a sus actitudes sobre la identidad de género, ubicando en la categoría de igualdad de género, por lo que se infiere que, esta parte de los participantes tienen actitudes favorables hacia la igualdad de género, existiendo una equidad en cuando al rol que desempeñan socialmente según su sexo, y del mismo modo, tiene menos actitudes de discriminación frente al otro sexo o en relación a cuestiones de género (Tabla 2).

Tabla 2

Identidad de género

\begin{tabular}{lll}
\hline $\begin{array}{l}\text { Identidad de } \\
\text { genero }\end{array}$ & $\mathrm{F}$ & $\%$ \\
\hline Sexista & 30 & 25 \\
Adaptativa & 54 & 45 \\
Igualitaria & 36 & 30
\end{tabular}




\begin{tabular}{|c|c|c|}
\hline Total & 120 & 100.0 \\
\hline Sociocultural & $\mathrm{F}$ & $\%$ \\
\hline Sexista & 32 & 26.7 \\
\hline Adaptativa & 49 & 40.8 \\
\hline Igualitaria & 39 & 32.5 \\
\hline Total & 120 & 100.0 \\
\hline Relacional & $\mathrm{F}$ & $\%$ \\
\hline Sexista & 45 & 37.5 \\
\hline Adaptativa & 73 & 60.8 \\
\hline Igualitaria & 2 & 1.7 \\
\hline Total & 120 & 100.0 \\
\hline Personal & $\mathrm{F}$ & $\%$ \\
\hline Sexista & 30 & 25 \\
\hline Adaptativa & 74 & 61.7 \\
\hline Igualitaria & 16 & 13.3 \\
\hline Total & 120 & 100.0 \\
\hline
\end{tabular}

De acuerdo con la revisión de los resultados de los instrumentos es necesario considerar que el empoderamiento de la mujer es predominantemente de un nivel medio en el $74.2 \%$ de las encuestadas, lo que muestra una realidad que va mejorando constantemente, es así como se vienen desarrollando mayor nivel de participación de las mujeres en escenarios de liderazgo, y también se encontró que el 23.3\% se ubicó en un nivel alto. Este resultado evidencia que los aspectos igualitarios percibidos han tenido una mejora, tales como las oportunidades de emprendimiento, el ejercicio de sus derechos y sobre todo el respaldo de la familia en las diversas acciones que toman (Tabla 3).

Tabla 3

Empoderamiento de la mujer

\begin{tabular}{lcc}
\hline $\begin{array}{l}\text { Empoderamiento } \\
\text { femenino }\end{array}$ & $\mathrm{F}$ & $\%$ \\
\hline Empoderamiento bajo & 17 & 14.2 \\
$\begin{array}{l}\text { Empoderamiento } \\
\text { medio }\end{array}$ & 89 & 74.2 \\
Empoderamiento alto & 14 & 23.3 \\
Total & 120 & 100.0 \\
\hline Participación & $\mathrm{F}$ & $\%$ \\
\hline
\end{tabular}




\begin{tabular}{|c|c|c|}
\hline Empoderamiento bajo & 51 & 42.5 \\
\hline $\begin{array}{l}\text { Empoderamiento } \\
\text { medio }\end{array}$ & 35 & 29.2 \\
\hline Empoderamiento alto & 34 & 28.3 \\
\hline Total & 120 & 100.0 \\
\hline Temeridad & $\mathrm{F}$ & $\%$ \\
\hline Empoderamiento bajo & 4 & 3.3 \\
\hline $\begin{array}{l}\text { Empoderamiento } \\
\text { medio }\end{array}$ & 41 & 34.2 \\
\hline Empoderamiento alto & 75 & 62.5 \\
\hline Total & 120 & 100.0 \\
\hline Influencias Externas & $\mathrm{F}$ & $\%$ \\
\hline Empoderamiento bajo & 28 & 23.3 \\
\hline $\begin{array}{l}\text { Empoderamiento } \\
\text { medio }\end{array}$ & 51 & 42.5 \\
\hline Empoderamiento alto & 41 & 34.2 \\
\hline Total & 120 & 100.0 \\
\hline Independencia & $\mathrm{F}$ & $\%$ \\
\hline Empoderamiento bajo & 5 & 4.2 \\
\hline $\begin{array}{l}\text { Empoderamiento } \\
\text { medio }\end{array}$ & 44 & 36.7 \\
\hline Empoderamiento alto & 71 & 59.2 \\
\hline Total & 120 & 100.0 \\
\hline Igualdad & $\mathrm{F}$ & $\%$ \\
\hline Empoderamiento bajo & 77 & 64.2 \\
\hline $\begin{array}{l}\text { Empoderamiento } \\
\text { medio }\end{array}$ & 40 & 33.3 \\
\hline Empoderamiento alto & 3 & 2.5 \\
\hline Total & 120 & 100.0 \\
\hline Satisfacción & $\mathrm{F}$ & $\%$ \\
\hline Empoderamiento bajo & 74 & 61.7 \\
\hline $\begin{array}{l}\text { Empoderamiento } \\
\text { medio }\end{array}$ & 17 & 14.2 \\
\hline Empoderamiento alto & 29 & 24.2 \\
\hline Total & 120 & 100.0 \\
\hline Seguridad & $\mathrm{F}$ & $\%$ \\
\hline Empoderamiento bajo & 48 & 40 \\
\hline $\begin{array}{l}\text { Empoderamiento } \\
\text { medio }\end{array}$ & 69 & 57.5 \\
\hline
\end{tabular}




\begin{tabular}{lcc} 
Empoderamiento alto & 3 & 2.5 \\
Total & 120 & 100.0 \\
\hline
\end{tabular}

Nota: Aplicación del SPSS26

Es preciso mencionar que se aplicó Pearson por cuanto la prueba de normalidad efectuada por Kolmogorov Smirnov ( $>50$ sujetos) obtuvo una distribución normal con un coeficiente de .605 para identidad de género y .058 para empoderamiento. En tal sentido, después de un análisis de las variables mediante la aplicación de la correlación de Pearson se ha identificado que existe relación entre las mismas ( $\mathrm{p}<.05 ; \mathrm{r}=.747)$, además la relación es positiva y alta, razón por la que se corrobora la hipótesis que se ha planteado (Ver tabla 4).

Tabla 4

Correlaciones

\begin{tabular}{clr}
\hline & & $\begin{array}{c}\text { Empoderamiento } \\
\text { de la mujer }\end{array}$ \\
\hline \multirow{2}{*}{ Identidad de } & Correlación de & $.747^{* *}$ \\
genero & Pearson & \\
& Sig. (bilateral) & 0.000 \\
& $\mathrm{~N}$ & 120 \\
& Correlación de & $.805^{*}$ \\
& Pearson & \\
& Sig. (bilateral) & 0.000 \\
& $\mathrm{~N}$ & 120 \\
& Correlación de & $.769^{*}$ \\
Sociocultural & Pearson & \\
& Sig. (bilateral) & 0.001 \\
& $\mathrm{~N}$ & 120 \\
& Correlación de & $.743^{*}$ \\
Relacional & Pearson & \\
& Sig. (bilateral) & 0.001 \\
& $\mathrm{~N}$ & 120 \\
**. La correlación es significativa en el nivel 0.01 (bilateral). & \\
Nota: Aplicación del SPSS26 &
\end{tabular}

La prueba de correlación de Pearson (Tabla 4) evidencia la relación entre las dimensiones y variables respectivamente, alcanzado de esta manera la aceptación de las hipótesis planteadas autoconcepto ( $\mathrm{p}<.05, \mathrm{r}=.805)$, roles de género $(\mathrm{p}<.05, \mathrm{r}=.769)$ y relaciones con los demás $(\mathrm{p}<.05$; $\mathrm{r}=.743$ ), es así que en medida que el individuo vaya incrementando aspectos de autoconcepto a 
nivel perceptivo y vaya desligando factores establecidos de roles a nivel familiar, aspectos educativos el empoderamiento podrá ir tomando mayor relevancia en el día a día.

\section{Discusión}

Desde un análisis de la realidad observada, se aprecia una relación entre las variables evaluadas ( $\mathrm{p}<.05 ; \mathrm{r}=.747$ ), lo cual corrobora la hipótesis planteada, en este sentido se puede afirmar que mientras mayor sea el puntaje de la variable empoderamiento femenino habrá mayor percepción de igualdad de género, que de acuerdo con el instrumento empleado, los individuos mostraran una actitud de igualdad ante los roles de género; a razón de que no existen otras investigaciones que aborden la relación entre ambas variables, se realiza un análisis a nivel teórico, para ello es importante definir cada una de las variables, Gauché y Lovera (2018), expresan que la identidad de género es una identidad que se adquiere mediante el aprendizaje a lo largo de los primeros años de vida, la construcción de la identidad de género forma parte de la vivencia interna e individual que cada sujeto experimenta por sí mismo; en cuanto a la variable de empoderamiento femenino, Ruiz, Turnbull y Cruz (2016), manifiestan que el empoderamiento femenino refiere a la capacidad de toma de decisiones que posee una mujer, tales decisiones promueven su autonomía y le hacen sentir que tiene dominio y poder sobre su propia vida.

Por otro lado, existen diversos organismos y autores que hacen referencia a estas dos variables, como por ejemplo el Federal Ministry for Europe (2017), afirma que el empoderamiento no solo requiere un aumento en la participación de las mujeres, sino, es necesario para cambiar las barreras estructurales con el fin de cambiar las normas sociales y culturales, políticas y relaciones en nuevas formas que permitan a mujeres y hombres asumir nuevos roles, desarrollando una identidad de género orientada a hacia la igualdad, para este organismo, el desarrollo del empoderamiento femenino tiene un gran impacto sobre la formación de la identidad de género de las mujeres, ya que, como se vio en la definición de las variables, la identidad de género es formada por la interacción que la persona tiene con su medio o entorno, en tal sentido, en un entorno donde la mujer tiene una participación donde refleja su empoderamiento reforzará sus niveles de identidad de género hacia una de mayor igualdad entre roles, existiendo mayor equidad; para Andrades, Palacio y Blanco (2019), el empoderamiento femenino es fundamental ya que es una poderosa herramienta para el desarrollo de habilidades y cualidades en las mujeres, con ella, la percepción de género femenino puede ir cambiando hacia una actitud de mayor igualdad. 
Realizando un análisis sobre las dimensiones, se encontró que, existe una relación muy alta y significativa entre el empoderamiento de la mujer y la dimensión personal ( $\mathrm{r}=.805)$, de este resultado se puede inferir que, un mayor empoderamiento tiene un enorme efecto sobre la capacidad de elegir en las mujeres, tal como lo mencionan García et al. (2010), al referirse que esta dimensión comprende las preferencias y elecciones de las mujeres, así como las aspiraciones vitales que puedan tener, y según Charlier et al. (2007), el empoderamiento actúa sobre el fortalecimiento del autoestima, la confianza y la capacidad de elegir el rumbo de su destino; permitiendo a las mujeres mayor capacidad en la toma de sus decisiones, permitiéndoles ir en búsqueda de las aspiraciones que puedan tener (Ruiz, Turnbull y Cruz, 2016), de esta manera se entiende a nivel teórico que el empoderamiento influye sobre la dimensión personal.

Respecto a la asociación entre el empoderamiento y la dimensión sociocultural, se encontró una correlación positiva alta ( $\mathrm{r}=.769)$, esta dimensión se define como el reparto equitativo entre varones y mujeres de los deberes en el hogar (García et al., 2010), en este sentido, el empoderamiento ayuda a que las mujeres tengan una mayor participación sobre las decisiones en el hogar y a la contribución en los ingresos económicos que estas puedan generar (Casique, 2006); así mismo, Charlier et al. (2007), afirman que, el empoderamiento posibilita un cambio en las relaciones de género para las diferentes áreas de la vida, como la sociocultural; estos aportes teóricos sustentan la manera en como el empoderamiento femenino tiene efecto sobre la dimensión sociocultural.

Finalmente, se tuvo un nivel de relación alto y positivo para la variable empoderamiento y la dimensión relaciones ( $\mathrm{r}=.743$ ), esta dimensión se relaciona con todos los aspectos asociados al trato igualitario que reciben las mujeres a nivel social, donde tienen un lugar como lideresas dentro de su comunidad, y también va asociado a las situaciones de violencia de las que ellas puedan ser víctimas (García et al., 2010), el efecto que tiene el empoderamiento sobre esta dimensión, se sustenta por la mencionado por Charlier et al. (2007), quien señala que el empoderamiento femenino permite un cambio a nivel político en beneficio de las mujeres, lo que favorece que ellas sean reconocidas y puedan ocupar cargos de lideresas en su comunidad; esto mismo es expresado por Casique (2006), quien también afirma que el empoderamiento contribuye con un trato más igualitario a nivel político entre varones y mujeres.

Finalmente, Las Naciones Unidas (2006), señala que, el empoderamiento es necesario para para que las mujeres dejen de tener una posición subordinada en la sociedad, lo que las hace un 
blanco fácil para ser víctimas de violencia, por otro lado, el empoderamiento dota a las mujeres de una mayor libertad para el pleno ejercicios de sus derechos, lo que contribuye a prevenir cualquier acto de violencia o discriminación hacia ellas, y posibilita también un trato más igualitario con sus pares varones en la sociedad; de esta manera se sustenta a nivel teórico la relación entre la variable empoderamiento femenino y la dimensión relaciones.

Para sintetizar, se afirma que, promover el empoderamiento en las mujeres contribuirá a una mejora a nivel personal, sociocultural y relacional. Por ende, mediante un análisis de la realidad estudiada se observa que los instrumentos aplicados demuestran que la identidad de género tiene un nivel medio prevalentemente en el $45.0 \%$ de la muestra, de este resultado se infiere que, en su mayoría, las encuestadas presentan actitudes que se adaptan a las circunstancias, pudiéndose mostrar a favor o no de la igualdad de género dependiendo de la situación se vio un nivel alto en el $30.0 \%$ de las encuestadas, de esto se infiere que, una tercera parte de las encuestadas tiene una posición de igualdad en relación a los roles de género entre varones y mujeres; estos resultados se asemejan a los valores encontrados por Boza (2019) en su investigación, donde el 69\% de las encuestas tenían un nivel promedio de identidad de género, y el 26.0\% un nivel alto.

En relación a la variable empoderamiento de la mujer, se encontró que el $74.2 \%$ de la muestra se ubicó en un nivel medio de empoderamiento, de esto se infiere que, la gran mayoría de mujeres encuestas aún necesitan desarrollar sus cualidades y potencialidades para ubicarse en mejores niveles de empoderamiento, ya que un nivel medio supone que ellas aún se encuentran en un proceso de mejora; además, se encontró que el 23.3\% de las encuestas se ubican en un nivel alto de empoderamiento, lo que supone que, este porcentaje de mujeres siente que tiene control sobre sus vidas, y se sienten capaces de tomar sus propias decisiones y elegir el camino a seguir. Por otro lado, estos resultados se asemejan con lo encontrado por Boza (2019), ya que en su estudio encontró que el 68.6\% de las mujeres que encuesto presentaban un nivel promedio de empoderamiento, y el $22.9 \%$ se mantuvo en un nivel alto.

En el contexto social, realizar la investigación en la asociación de vecinos Quinta Elena presentó limitaciones al momento de la aplicación de las encuestas, ya que este proceso fue realizado en medio de la crisis sanitaria por Covid 19, se suma a esto el tiempo que la mayoría de sus habitantes dedican al cuidado del hogar y sus familias, acoplarse a un horario adecuado para la realización de las encuestas sin que ellas puedan sentir que estaban descuidando esta área de su vida fue un gran desafío, a su vez se observó temor al hablar de ciertos puntos relacionados al 
empoderamiento femenino y como se sentían al cumplir su rol de mujeres ante la sociedad, tema que para algunas de las encuestadas era poco conocido.

\section{Conclusión}

Durante la realización del presente estudio se dieron hallazgos en torno a la identidad de género y el empoderamiento femenino en las habitantes de la asociación de vecinos de Quinta Elena, los cuales arrojaron resultados proporcionales, llegando a la conclusión de que, a mayores niveles de empoderamiento, mayor será el nivel de identidad de género en las mujeres las cuales se orientan hacia actitudes de igualdad y/o equidad de género. En última instancia, se considera necesario que la aplicación de los instrumentos pueda ser aplicado en una población mucho mayor a fin de efectuar análisis comparativos en diversas realidades.

\section{Referencias}

Agencia Peruana de Noticias. (21 de mayo de 2018). Perú mejoró en ranking del índice global de brecha de género. Andina. https://andina.pe/agencia/noticia-ministra-de-mujer-perumejoro-ranking-del-indice-global-brecha-genero-710735.aspx

Alvarado, A. (2016). La imagen de la mujer de élite en la costa norte del Perú a través de las crónicas de Indias. Lex: Revista de la Facultad de Derecho y Ciencia Política de la Universidad Alas Peruanas, 14(18), 379-398. http://dx.doi.org/10.21503/lex.v14i18.1249

Andrades, N., Palacio, A., \& Blanco, A. (2019). Empoderamiento femenino e igualdad de género en las organizaciones. Liderazgo Estratégico, 9(1), 140-148. https://revistas.unisimon.edu.co/index.php/liderazgo/article/download/3809/4293

Ballon, P., \& Yalonetzky, G. (2018). Introduction to Special Section: Quantitative Approaches to the Measurement and Analysis of Female Empowerment and Agency. The Journal of Development Studies. 54(8), 1279-1283, https://doi.org/10.1080/00220388.2017.1414191

Batliwala, S. (1997). El significado del empoderamiento de las mujeres: Nuevos conceptos desde la acción. Poder y empeoramiento de las mujeres. 187-2011. http://www.congresoed.org/wp-content/uploads/2014/10/D4_Batliwala_1997.pdf

Boza, G. (2019). Empoderamiento de la mujer en las Organizaciones Sociales de Base del Primer Nivel de los Clubes de Madres - Distrito de Carmen Alto, Huamanga, Ayacucho. (Tesis de pregrado), Universidad Inca Garcilaso de la Vega, Perú. http://repositorio.uigv.edu.pe/bitstream/handle/20.500.11818/4581/TRABSUFICIENCIA _BOZA_GRACIELA.pdf?sequence=1\&isAllowed=y

Bandura, A., \& Walters, R. (1963). Social learning and personality development. New York: Rinehart and Winston. 
Calderon, J. (2017). Identidad de género y empoderamiento de la mujer en la asociación de mujeres indígenas de Melgar. (Tesis de pregrado), Universidad Nacional del Altiplano, Perú.

http://repositorio.unap.edu.pe/bitstream/handle/UNAP/10163/Calder\%c3\%b3n_Quispe_J ulia_Roxana.pdf?sequence $=1 \&$ isAllowed $=y$

Charlier, S., Caubergs, L., Drory, E., Kiteel, F., Kakiba, E., Staes, V., Ravesloot, S., Malpas, N., Smets, K., \& Grolet, S. (2007). El proceso de empoderamiento de las mujeres. Guía metodológica. Comisión de Mujeres y Desarrollo. https://dhls.hegoa.ehu.eus/uploads/resources/4668/resource_files/proceso_empoderamient o_mujeres_CFD.pdf

Casique, I. (2006). ¿Cuándo puedo decir no? Empoderamiento femenino y sexo no deseado en México. Estudios Demográficos y Urbanos, $\quad 21(1), \quad 49.81$. https://www.redalyc.org/articulo.oa?id=31200102

Duarte, J. y García, J. (2016). Igualdad. equidad de género y feminismo. una mirada histórica a la conquista de los derechos de las mujeres. Revista CS, 18, 107-158. http://dx.doi.org/10.18046/recs.i18.1960

Espinoza, E. (2020). El rol de las redes sociales y el empoderamiento de las mujeres en medicina. Revista Peruana de Medicina Experimental y Salud Pública. 37(1). 136-141. http://dx.doi.org/10.17843/rpmesp.2020.371.5092

Farías, L. y Cuello, V. (2018). Percepción y autopercepción de la identidad e igualdad de género en estudiantes universitarios de la Región de Valparaíso a través de la publicidad. Revista de Comunicación, $17(1)$, 155-165. http://www.scielo.org.pe/pdf/rcudep/v17n1/a09v17n1.pdf

Fundación Romero (08 de marzo de 2020). MasterCard: Perú entre los países con mayor empoderamiento de mujeres. PQS. https://www.pqs.pe/emprendimiento/mastercard-peruentre-los-paises-con-mayor-empoderamiento-de-mujeres

Federal Ministery for Europe (2017). Gender Equality and the Empowerment of Women and Girls. $\left(1 \mathrm{e}^{\mathrm{ra}}\right.$ ed.), Viena. https://www.entwicklung.at/fileadmin/user_upload/Dokumente/Publikationen/Leitlinien/ Englisch/PD_Gender_2017_EN.pdf

Freud, S. E. (1957). The ego and the id. London: Hogarth.

García, R., Rebollo, M., Buzón, O., González-Piñal, R., Barragán, R., \& Ruiz, E. (2010). Actitudes del Alumnado hacia la igualdad de género. Revista de Investigación Educativa, 28(1), 217232.

https://digitum.um.es/digitum/bitstream/10201/44935/1/Actitudes\%20del\%20alumnado\% 20hacia\%20la\%20igualdad\%20de\%20genero.pdf 
Gauché, X.; \& Lovera, D (2018). Identidad de género de niños, niñas y adolescents: Una cuestión de derechos. Revista Lus et Praxis, 25(2), 359-402. DIO: http://dx.doi.org/10.4067/S071800122019000200359

Hernández, J.; \& García, R. (2008). Instrumento para medir el empoderamiento de la mujer. (1 $1^{\text {era }}$ ed.), Universidad Juárez Autónoma de Tabasco. http://cedoc.inmujeres.gob.mx/documentos_download/101158.pdf

Kasey, W., Champlin, S., Shelton, S., Sterbenk, Y., \& Poteet, M. (2019). Selling Feminism: How Female Empowerment Campaigns Employ Postfeminist Discourses. Journal of Advertising, 49, 18-33. http://dx.doi.org/10.1080/00913367.2019.1681035

Leiva, J., Martín, V. \& Vila, E. (2015). Género. Educación y Convivencia. Dykinson, 28(2), 304315. http://dx.doi.org/10.2307/j.ctt1k233dj

Lynn, D. B. (1965). Parental and sex role identificaction: A theorical formulation. Berkeley, CA: McCutchan.

Mischel, W. (1966). A social learning view of sex differences. Stanford, CA: Stanford University Press.

Nigam, A. K., Srivastava, R., Kulshrestha, A. C., \& Kumar, K. (2020). Female Empowerment: A Life-Cycle Analysis. Cambridge Scholars Publishing. https://www.cambridgescholars.com/female-empowerment

Ñaupas, H., Mejía. E., Novoa, E. y Villagómez, A. (2014). Metodología de la investigación. (4 ed.). Ediciones de la U. https://n9.cl/317p6

Naciones Unidas (2006). Poner fin a la violencia contra la mujer. De las palabras a los hechos. Publicación de las Naciones Unidas. https://www.un.org/womenwatch/daw/vaw/publications/Spanish\%20study.pdf

Otzen, T. y Manterola, C. (2017). Técnica de muestreo sobre una población a estudio. International Journal of Morphology. 35(1), 227-232. http://dx.doi.org/10.4067/S071795022017000100037

Padilla, N., y Cruz, C. (2019). Salud emocional y sexual: Una aproximación para el ciudado y empoderamiento femenino. ( $1^{\circ}$ ed.) México: Universidad Iberoamericana. https://n9.cl/8j93w

Ramos, C. (1999). Identidad de género. Estudios de género. México. 1 (10). http://148.202.18.157/sitios/publicacionesite/pperiod/laventan/Ventana10/ventana107Carmen.pdf

Ros, T., \& Lidón, B. (2018). La experiencia del empoderamiento en mujeres mayores de 65 años: Estudio cualitativo. Gerokomos. 3-8. http://scielo.isciii.es/scielo.php?script=sci_arttext\&pid=S1134-928X2018000100003 
Ruiz, P., Vargas, S., \& Clausen, J. (2018). Empoderar para incluir: Análisis de las múltiples dimensiones y factores asociados al empoderamiento de las mujeres en el Perú a partir del uso de una aproximación de metodologías mixtas. Instituto Nacional de Estadística e informática.

https://www.inei.gob.pe/media/MenuRecursivo/investigaciones/empoderamiento-de-lasmujeres.pdf

Ruiz, A., Turnbull, B., \& Cruz, C. (2016). Construcción del concepto de empoderamiento en el hogar en un grupo de mujeres del Estado de México (México). Enseñanza e Investigación en Psicología, 21(53), 153-160. https://www.redalyc.org/pdf/292/29248181005.pdf

Rosazza, G. (2019). Empoderamiento femenino y la violencia en usuarias del Centro de Emergencia Mujer en la ciudad de Huaraz. periodo 2019 (Tesis de pregrado). Universidad Nacional José Faustino Sánchez Carrión. Perú. http://repositorio.unjfsc.edu.pe/bitstream/handle/UNJFSC/3311/Rosazza\%20Monta\%C3 \%B1ez\%20y\%20Piscoya\%20Chavarri.pdf?sequence=1\&isAllowed=y

Rocha, T. (2009). Desarrollo de la Identidad de Género desde una Perspectiva Psico-SocioCultural: Un Recorrido Conceptual. Revista Interamericana de Psicología, 43(2), 250-259. https://www.redalyc.org/pdf/284/28412891006.pdf

Silvestre, M., Royo, R., y Escudero, E. (2014) El empoderamiento de las mujeres como estrategia de intervención social. España: Universidad de Deusto. https://dialnet.unirioja.es/servlet/libro?codigo=738433

Simons, J. (2020). Sexual and Gender Minority Identity Development: Recommendations for School Counselors. Revista de Consejería Escolar, 18(20). https://eric.ed.gov/?id=EJ1258650

Torres, C. (2019). ¿Cómo empoderar a la mujer peruana? Artículo Gestión. https://gestion.pe/blog/te-lo-cuento-facil/2019/07/como-empoderar-a-la-mujerperuana.html/?ref=gesr

Urcelay, R. (2014). El empoderamiento de las mujeres. La Ruta para una vida equitativa y segura. (Tesis de posgrado). Universitat Jaume I, España. http://repositori.uji.es/xmlui/bitstream/handle/10234/107662/TFM_2013_urzelaiR.pdf?se quence $=1 \&$ is Allowed $=\mathrm{y}$ 